\title{
COVID-19 and Asthma, Lucky or Unlucky?
}

\author{
Jiu-Yao Wang ${ }^{1}$, Ruby Pawankar ${ }^{2}$, Hui-Ju Tsai ${ }^{3}$, and Shih-Hsin Wu ${ }^{4}$ \\ ${ }^{1}$ College of Medicine, National Cheng Kung University \\ ${ }^{2}$ Nippon Medical School \\ ${ }^{3}$ National Health Research Institutes \\ ${ }^{4}$ Tzu Chi University
}

June 1, 2020

\section{Main text:}

"Misfortune might be a blessing in disguise."

Tao-Te-Ching (Book of the Way) by Lao-Tzu, 350 B.C.E

Since its first report in Wuhan, China, in December 2019, the novel pandemic COVID-19 caused by the severe acute respiratory syndrome coronavirus 2 (SARS-CoV2 virus), has rampaged throughout the world. Approximately $80 \%$ of the COVID-19 patients manifest with only mild upper respiratory symptoms, $10-15 \%$ develop bilateral infiltrated pneumonia, and 5 to $10 \%$ of the severe cases progress to develop Acute Respiratory Disease Syndrome (ARDS) and multiple organs failure by the 'cytokine releasing storm' (CRS). ${ }^{1}$ Older age, male gender, chronic cardiovascular diseases and metabolic syndrome, such as obesity, hyperlipidemia, and diabetes are among the risk factors associated with severe COVID-19. ${ }^{2}$ Asthmatics are known to be more frequently infected and at a greater risk of developing more severe outcomes with respiratory/common cold viral infections, particularly in those with poorly controlled asthma. ${ }^{3}$ Moreover, there is a deficiency and delay of the production of type I and type III interferons in the lung cells of asthmatic patients, potentially more favorable for SARS-CoV2 infection that depends on a hampered innate anti-virus immune response in the respiratory tract. ${ }^{3}$ However, recent reports have accumulated evidences that the prevalence of allergic diseases and asthma in patients with COVID-19 is lower than expected and underrepresented among other comorbidities and risk factors of the severe form of COVID-19. ${ }^{4-6} \mathrm{Li}$ et al. ${ }^{5}$ reported that the prevalence of asthma in patients with severe COVID-19 ( $\mathrm{n}=269,49.1 \%$ of total 548 patients) was $0.9 \%$, markedly lower than that in the adult population of Wuhan. Moreover, none of the children (below 12 years of age) infected with SARS-CoV2 at the Wuhan Children's Hospital had underlying asthma. ${ }^{6}$ These findings on the low prevalence of asthma may be confounded by a sampling bias (all hospitalized patients) in the reported cases, underdiagnoses, or lack of recognition of asthma in those with COVID-19. In fact, lack of surveys of the confirmed COVID-19 cases in the general population is a major limiting factor in answering the question whether chronic respiratory diseases, such as asthma, are key risk factors of SARS-CoV2 infection. ${ }^{4}$

However, the interesting question to ask is why asthmatic patients did not have an increased exacerbation of asthma or became more prone to the severe form of COVID-19 after being infected with SARS-CoV2? We, therefore, propose the following plausible mechanisms in asthmatic patients that may have effects in the determination of the susceptibility and severity regarding SARS-CoV2 infection. Firstly, coronavirus recognition and infection is dependent on the cellular receptors, the angiotensin converting enzyme 2 (ACE2) for ducking spike protein of SARS-Cov2, and transmembrane protease serine 2 (TMPRSS2) to cleave the ducked spike protein for virus entry by membrane fusion. ${ }^{7}$ Lung cells that co-express these two molecules include nasal goblet secretory cells, bronchial transient secretory cells, and alveolar type II pneumocytes. ${ }^{8}$ The gene expression levels of ACE2 and TMPRSS2 are influenced by the genetic variants of the host, microbial infec- 
tions, and induced by innate immune response, such as the production of interferons and mucins. ${ }^{8}$ It has been found that SARS-COv2 virus can hijack and enhance this innate anti-virus defense interferon production to induce ACE2, a human interferon stimulated gene (ISG), expression in the other uninfected cells, and promote its potential transmission and spread. ${ }^{8}$ While in asthma patients, the expression of these two molecules in the respiratory epithelial cells for SARS-CoV2 infection are determined by the age, gender, comorbidity and type 2 allergic inflammation. Jackson et al. found asthma and respiratory allergy are associated with reduced ACE2 expression in airway cells based on their patient cohorts. ${ }^{9}$ Moreover, ACE2 expression in the lower airway epithelium was lower in the post-allergen challenge samples, and its levels of expression was also significantly inversely associated with type 2 immune inflammation biomarkers. ${ }^{9}$ In contrast, in an analysis of the nasal airway epithelial transcriptome in 695 children, Sajuthi et al. ${ }^{10}$ demonstrated that TMPRSS2 is part of a mucus secretory network, highly upregulated by type 2 allergic inflammation mainly by interleukin-13. Peters et al. ${ }^{11}$ from the same group, found the gene expression for ACE2 and TMPRSS2 in the cells obtained from induced sputum did not differ between healthy and asthmatics. Instead, a higher expression of ACE2 and TMPRSS2 in males, African Americans, and patients with diabetes mellitus provides rationale for monitoring these asthmatic subgroups for poor COVID19 outcomes. ${ }^{11}$ Therefore, these reports did not describe convincing information as to whether the asthmatic patients had a lower expression of ACE2 and serine protease TMPRSS2 for SARS-CoV2 based on their allergic status and/or chronic lung inflammation. Particularly, these studies lacked information on the effect of SARS- CoV-2- specific analysis and observations in asthmatic patients to be able to reach a conclusion in the real world.

Secondly, viral load and immune response of the host determines the final outcome and/or the severity of ARDS and multiple organ failures in COVID-19 patients. At the initial phase, antivirus innate immune response determine the clearance of virus by the production of Type I/III interferons of infected cells. Lack of or lowered production of IFN $\alpha 2$ and IFN $\lambda$, and high viral loads are frequently found in the severe cases that deteriorated into needing ICU care. ${ }^{12}$ At the second phase of COVID-19 infection, aberrant host immune response by infected macrophages that lead to cytokines releasing storm, and $\mathrm{T}$ lymphocytes depletion due to the prolonged viral infection are the two major determining factors for a final clinical outcome. ${ }^{12}$ For asthmatic patients, the innate immune response to COVID-19 infection may be impaired due to lower levels of IFN $\gamma$ in their bronchial epithelial cells, but it may also turn into favor for reducing ACE2 expression, which is depended on IFN $\gamma$ production. ${ }^{8}$ In addition to IFNs, there are other molecules of innate immunity in the respiratory tract that may also have anti-viral functions, such as mannose binding lectin (MBL), and surfactant protein A (SP-A) and D (SP-D) that are produced by alveolar type 2 cells in the lung, which are also the largely infected by SARS coronavirus. These molecules, MBL and SP-D, found in higher concentrations in the Broncho-alveolar lavage fluids (BALF) of patients with asthma and respiratory allergy and are increased due to chronic inflammation, have been identified to bind spike protein of SARS coronavirus, and inhibit its binding to ACE2 cellular receptor, and thereby able to protect the alveolar macrophages from virus-induced activation. ${ }^{13,14}$

Recently, it is found that long-term boosting of the innate immune responses, also termed 'trained immunity', by prior microbial infection or certain live vaccines, induces heterologous protection against infections, through epigenetic, transcriptional and functional reprogramming of innate immune cells. ${ }^{15}$ Experimental studies have demonstrated that alveolar or lung macrophages can also undergo long-term reprogramming after infections. For example, gamma herpes virus infection can protect against an allergic response in experimental asthma, ${ }^{16}$ while adenovirus infection-induced remodeling in alveolar macrophages and subsequently induced more pronounced anti-bacterial immunity. ${ }^{17}$ This trained immunity in the myeloid cells and alveolar macrophages of asthmatics may provide anti-viral immunity in specific organs such as the lungs. Although this hypothesis has not been validated in patients with COVID-19, clinical trials to booster trained innate immunity by BCG vaccination to protect against COVID-19 have been initiated in several countries. ${ }^{18}$

Finally, we highlight that therapeutic medications and biologics used for asthma control may have some beneficial pharmacological effects in COVID-19 infections. From in-vitro models, inhaled corticosteroids alone or in combination with bronchodilators have been shown to suppress coronavirus replication and cytokine production. ${ }^{19}$ Asthmatic patients using inhaled corticosteroids (ICS) demonstrated lower expression 
of ACE2 and TMPRSS2 in their bronchial epithelial cells. ${ }^{11}$ There is a clinical report of the improvement in three COVID-19 patients after using inhaled ciclesonide, although this study did not have proper controls. ${ }^{20}$ Before the outbreak of COVID-19, clinical observation in children with severe asthma who received anti-IgE monoclonal antibody (omalizumab) have shown decreased duration of human rhinovirus (HRV) infections, viral shedding, and risk of HRV-related illnesses compared with guideline-driven care alone. ${ }^{21}$ In vitro, omalizumab attenuated plasmacytoid dendritic cell (pDC) FceRI $\alpha$ protein expression while simultaneously augmenting pDC IFN- $\alpha$ responses to HRV and influenza virus. ${ }^{22}$ Together, these findings provide direct evidence that blocking IgE decreases susceptibility to respiratory viral illnesses through enhanced IFN- $\alpha$ responses in pDCs. Whether this anti-virus effect by anti-IgE treatment also includes infection with coronavirus and SARS-CoV2 is an open question that remains to be verified. More interestingly, azithromycin combined with hydroxychloroquine in an open-label non-randomized clinical trial for COVID-19 patients, has significant decreased the viral load after six days of treatment compared to untreated controls. ${ }^{23}$ Azithromycin has been demonstrated to reduce the frequency of asthma exacerbation and improve the quality of life of asthmatic adults and preschool children with asthma that was not adequately controlled on standard inhaler therapy. ${ }^{24}$ Although the mechanisms of this anti-inflammatory effect in asthma is still not well defined, some studies presented a reduction of IL-6 levels after azithromycin treatment. ${ }^{25}$ Moreover, a relevant study from Gielen and co-workers demonstrated that azithromycin augments IFN- $\beta$ and IFN- $\lambda$ production and decreasing rhinovirus replication and release from rhinovirus-infected human bronchial epithelial cells in vitro. ${ }^{26}$ Hence, it is suggested that azithromycin may have protective effects in reducing SARS-CoV-2 invasion by interfering with ligand/CD147 receptor interactions, a novel SARS-CoV2 cellular receptors beside ACE2, and decreasing the expression of some metalloproteinase (downstream to CD147) in primary human bronchial epithelial infected with rhinovirus. ${ }^{27}$ However, controlled clinical trials using azithromycin to treat patients with COVID-19 (not involving asthmatic subjects) are now registered in several countries with results still pending.

In summary, we have seen a new zoonotic coronavirus, SARS-CoV2, infection that has had a devastating effect on the host immunity via the inhibition of interferons leading to aberrant innate immune response, macrophages inflammation in cytokine releasing storm, and exhaustion of the cellular immunity of $\mathrm{T}$ lymphocytes. ${ }^{28}$ In contrast, due to chronic and sustained type 2 immune inflammation in the lungs of asthmatic patients, or fortunately by the medications they use for asthma control, it seems asthma may not be a major confounding disease in COVID-19 infection, and this unexpected phenomenon may throw a new light in finding therapies or preventative strategies for SARS-CoV2 (Figure 1 ). We still need a more comprehensive and in-depth immune analysis of SARS-CoV2 infection in the coming days to explore this hypothesis. However, all standard asthma therapies, whether inhaled steroids, combination of inhaled steroid plus long acting bronchodilator therapies, or monoclonal antibodies like omalizumab and azithromycin, should be continued to be used to optimize asthma control as recommended by all medical societies, for they can not only substantially reduce the risk of asthma exacerbation, but also will reduce risks and severe outcomes of COVID-19.

\section{Acknowledgements:}

JYW is supported by the Center for Allergy and Clinical Immunology Research (ACIR), Research and Service Headquarter, and in part by the Headquarters of University Advancement, National Cheng Kung University, Tainan, Taiwan.

\section{Conflict of Interests statement:}

All authors declare no conflict of interests

\section{References}

1. Guan WJ, Ni ZY, Hu Y, Liang WH, Ou CQ, He JX, et al. Clinical characteristics of coronavirus disease 2019 in China. N Engl J Med 2020; 382 :1708-1720.

2. Zhou F, Yu T, Du R, Fan G, Liu Y, Liu Z, et al. Clinical course and risk factors for mortality of adult inpatients with COVID-19 in Wuhan, China: a retrospective cohort study. Lancet 2020; 395 : 
1054-62.

3. Jartti T, Gern JE. Role of viral infections in the development and exacerbation of asthma in children. J Allergy Clin Immunol. 2017;140 :895-906.

4. Halpin DMG, Faner R, Sibila O, Badia JR, Agusti A. Do chronic respiratory diseases or their treatment affect the risk of SARS-CoV-2 infection? Lancet Respir Med. 2020;8 :436-438.

5. Li X, Xu S, Yu M, Wang K, Tao Y, Zhou Y, et al. Risk factors for severity and mortality in adult COVID-19 inpatients in Wuhan. J Allergy Clin Immunol. 2020 Apr 12. doi: 10.1016/j.jaci.2020.04.006.

6. Lu X, Zhang L, Du H, et al. SARS-CoV-2 infection in Children. N Engl J Med. 2020;382(17):16631665 .

7. Hoffmann M, Kleine-Weber H, Schroeder S, Krüger N, Herrler T, Erichsen S, et al. SARS-CoV-2 cell entry depends on ACE2 and TMPRSS2 and is blocked by a clinically proven protease inhibitor. Cell. 2020;181:271-280.

8. Ziegler CGK, Allon SJ, Nyquist SK, Mbano IM, Miao VN, Tzouanas CN, et al. SARS-CoV-2 receptor ACE2 is an interferon-stimulated gene in human airway epithelial cells and is detected in specific cell subsets across tissues. Cell. 2020 Apr 27. doi: 10.1016/j.cell.2020.04.035.

9. Jackson DJ, Busse WW, Bacharier LB, Kattan M, O'Connor GT, Wood RA, et al. Association of respiratory allergy, asthma and expression of the SARS-CoV-2 receptor, ACE2. J Allergy Clin Immunol. 2020 Apr 22. pii: S0091-6749(20)30551-0. doi: 10.1016/j.jaci.2020.04.009.

10. Sajuthi SP, DeFord P, Jackson ND, Montgomery MT, Everman JL, Cydney L. Rios CL, et al. Type 2 and interferon inflammation strongly regulate SARS- CoV-2 related gene expression in the airway epithelium. Preprint at bioRxiv https://doi.org/10.1101/2020.04.09.034454 (2020)

11. Peters MC, Sajuthi S, Deford P, Christenson S, Rios CL, Montgomery MT, et al. COVID-19 related genes in sputum cells in asthma: Relationship to demographic features and corticosteroids. Am J Respir Crit Care Med. 2020 Apr 29. doi: 10.1164/rccm.202003-0821OC.

12. Blanco-Melo D, Nilsson-Payant BE, Liu WC, Uhl S, Hoagland D, Møller R, et al. Imbalanced host response to SARS-CoV-2 drives development of COVID-19. Cell. 2020 May 13. doi: 10.1016/j.cell.2020.04.026.

13. Leth-Larsen R, Zhong F, Chow VT, Holmskov U, Lu J. The SARS coronavirus spike glycoprotein is selectively recognized by lung surfactant protein $\mathrm{D}$ and activates macrophages. Immunobiology. 2007;212 :201-11.

14. Zhou Y, Lu K, Pfefferle S, Bertram S, Glowacka I, Drosten C, et al. A single asparagine-linked glycosylation site of the severe acute respiratory syndrome coronavirus spike glycoprotein facilitates inhibition by mannose-binding lectin through multiple mechanisms. J Virol 2010;84 :8753-64

15. Netea MG, Joosten LA, Latz E, Mills KH, Natoli G, Stunnenberg HG, et al. Trained immunity: a program of innate immune memory in health and disease. Science. 2016;352 :aaf1098.

16. Machiels B, Dourcy M, Xiao X, Javaux J, Mesnil C, Sabatel C, et al. A gammaherpesvirus provides protection against allergic asthma by inducing the replacement of resident alveolar macrophages with regulatory monocytes. Nat Immunol 2017; 18 :1310-1320.

17. Yao Y, Jeyanathan M, Haddadi S, Barra NG, Vaseghi-Shanjani M, Damjanovic D, et al. Induction of autonomous memory alveolar macrophages requires $\mathrm{T}$ cell help and is critical to trained immunity. Cell 2018; 175 : 1634-1650.e17.

18. O'Neill LAJ, Netea MG. BCG-induced trained immunity: can it offer protection against COVID-19? Nat Rev Immunol 2020 May 11. doi: 10.1038/s41577-020-0337-y.

19. Yamaya M, Nishimura H, Deng X, Sugawara M, Watanabe O, Nomura K, et al. Inhibitory effects of glycopyrronium, formoterol, and budesonide on coronavirus HCoV-229E replication and cytokine production by primary cultures of human nasal and tracheal epithelial cells. Respir Investig. 2020;58 :155-168.

20. Iwabuchi K, Yoshie K, Kurakami Y, Takahashi K, Kato Y. Morishima T. COVID-19. Three cases improved with inhaled ciclesonide in the early to middle stages of pneumonia. 2020. http://www.kansensho.or.jp/uploads/files/topics/2019ncov/covid19_casereport_200310.pdf (accessed March 27, 2020; in Japanese). 
21. Esquivel A, Busse WW, Calatroni A, Togias AG, Grindle KG, Bochkov YA, et al. Effects of omalizumab on rhinovirus infections, illnesses, and exacerbations of asthma. Am J Respir Crit Care Med. 2017;196 :985-992.

22. Gill MA, Liu AH, Calatroni A, Krouse RZ, Shao B, Schiltz A, et al. Enhanced plasmacytoid dendritic cell antiviral responses after omalizumab. J Allergy Clin Immunol 2018;141:1735-43.e9.

23. Gautret P, Lagier JC, Parola P, Hoang VT, Meddeb L, Mailhe M, et al. Hydroxychloroquine and azithromycin as a treatment of COVID-19: results of an open-label non-randomized clinical trial. Int J Antimicrob Agents. 2020:105949.

24. Gibson PG, Yang IA, Upham JW, Reynolds PN, Hodge S, James AL, et al. Effect of azithromycin on asthma exacerbations and quality of life in adults with persistent uncontrolled asthma (AMAZES): a randomised, double-blind, placebo-controlled trial. Lancet. 2017;390 :659-68.

25. Bouwman JJM, Visseren FLJ, Bouter PK, et al. Azithromycin inhibits interleukin-6 but not fibrinogen production in hepatocytes infected with cytomegalovirus and chlamydia pneumoniae. J Lab Clin Med 2004;144:18-26

26. Gielen V, Johnston SL, Edwards MR. Azithromycin induces anti-viral responses in bronchial epithelial cells. Eur Respir J. 2010;36(3):646-54.

27. Ulrich H, Pillat MM. CD147 as a target for COVID-19 treatment: Suggested effects of azithromycin and stem cell engagement. Stem Cell Rev Rep. 2020 Apr 20. doi: 10.1007/s12015-020-09976-7.

28. Vabret N, et al, Immunology of COVID-19: current state of the science. Immunity 2020. https://doi.org/10.1016/j.immuni.2020.05.002

\section{Figure 1:}

The immuno-pathogenesis of COVID-19 and asthma

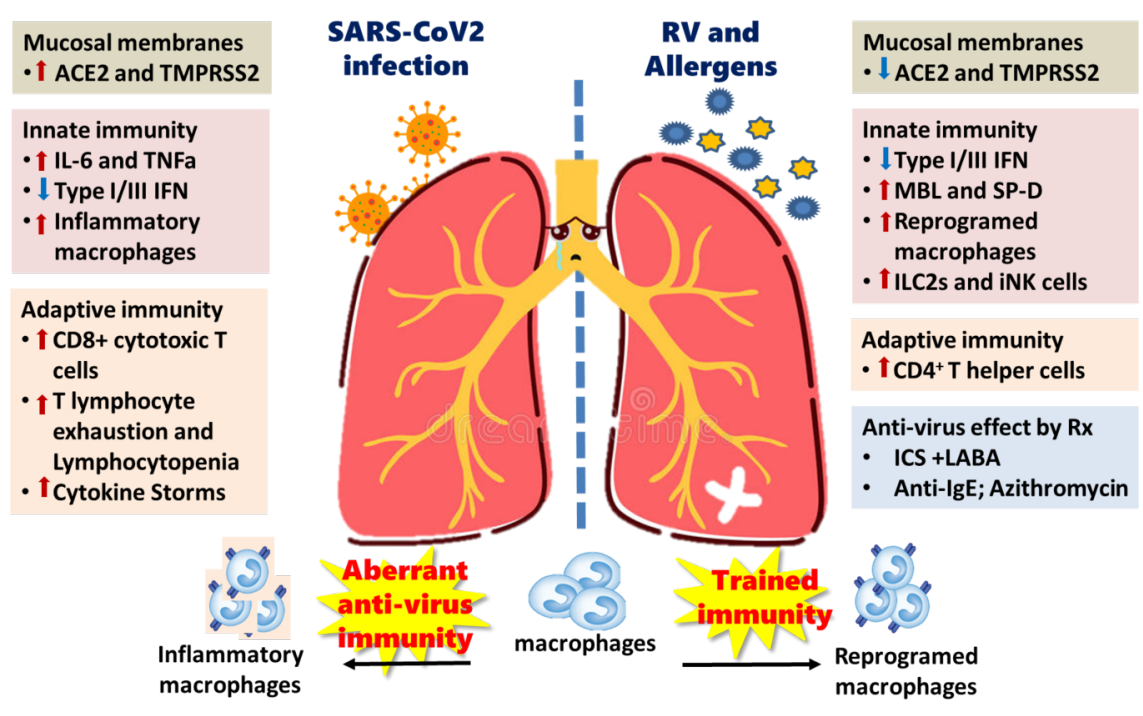

